\title{
ORIGINAI
}

\section{EPIDEMIOLOGÍA DEL CÁNCER INVASOR DE CÉRVIX EN EL ÁREA SANITARIA DE GIRONA DURANTE EL PERÍODO 1980-1989. REGISTRO POBLACIONAL DE CÁNCER DE GERONA}

\author{
Sánchez Garrido Victoria (1), Izquierdo Font Ángel (2,3), Beltrán Fabregat Miguel (3) y \\ Viladiu Quemada Pau $(1,2)$. \\ (1) Instituto Catalán de Oncología de Barcelona. \\ (2) Unidad de Epidemilogía y Registro del Cáncer. Instituto de Asistencia Sanitaria de Gerona. \\ (3) Servicio de Oncología del Hospital Santa Catalina de Gerona.
}

\section{RESUMEN}

Fundamento: Estudio descriptivo del cáncer invasor de cérvix en el Area Sanitaria de Gerona, a partir de los datos del Registro Poblacional de Cáncer.

Metodos: El estudio comprende el período de 1980 a 1989 y la población observada incluye a las mujeres residentes en el Area Sanitaria. Se ha realizado el cálculo de tasas de incidencia bruta, ajustada, truncada y acumulada, así como un análisis de tendencia, de distribución demográfica del tumor y un análisis de la supervivencia poblacional.

Resultados: La edad media de presentación del cáncer fue de $57 \pm 14$ años; el tipo histológico más frecuente el carcinoma escamoso; el $51 \%$ de los casos son mujeres nacidas en Cataluña, el resto son inmigrantes y extranjeras. La distribución de estadíos en el momento del diagnóstico fue: $36 \%$ casos locales, $34 \%$ regionales y $23 \%$ diseminados. Las tasas bruta, ajustada y truncada de incidencia en el período 1980-89 eran de 8.2, 6.2 y 13.7 casos $\times 10^{5}$ mujeres respectivamente. Según la tasa acumulada, una de cada 150 mujeres sufrirán este tumor antes de los 75 años. No se observa un incremento significativo de la tendencia; por el contrario, se aprecia una subincidencia significativa en zonas ruralcs. La probabilidad de supervivencia relativa a $\operatorname{los} 5$ años es de1 $48.5 \%$, no apreciándose diferencias significativas en función de la edad pero sí en función del estadío.

Conclusiones: En comparación con las cifras internacionales, Gerona es un área de baja incidencia de este tumor, no observándose una tendencia ascendente del mismo. La subincidencia en zonas rurales puede sugerir una diferente exposición a factores de riesgo, aunque hay que tener en cuenta aspectos como la accesibilidad a los servicios sanitarios, la definición de medio urbano y rural, y la presencia de movimientos migratorios. Por último, la menor supervivencia observada en comparación con otros países podría atribuirse a la presencia de estadíos más avanzados, lo que pone de relieve la importancia de la detección precoz de este tumor.

Palabras clave: Cánccr invasor de cérvix. Registro Poblacional de Cáncer. Incidencia. Supervivencia.

Correspondencia:

M. ${ }^{a}$ Victoria Sánchez Garrido.

Unitat de Prevenció i Control del Càncer

Institut Català d'Oncologia. Avda. Gran Vía s/n. km. 2,7.

08907 L'Hospitalet. Barcelona. FAX: (93) 2633556.
ABSTRACT

\section{Epidemiology of Cervical Invasive Cancer in the Gerona Health Area from 1980 to 1989. Cancer Population Records of Gerona}

Background: The goal of the present work is to carry out a descriptive study about the impact of invasive cervical cancer in the area of Gerona, Spain.

Methods: Using data from the population bascd cancer registry of Gerona for the period 1980-89, several incidence rates were calculated. Incidence trends, demographical distribution and survival according of different variables were analyzed.

Results: The average age in which the disease appears was $57 \pm 14$ years; the most frequent histological type was the squamous cell carcinoma; $51 \%$ of the cases were women born in Cataluña and the remaining percentage corresponds to women from others regions and foreigners. At the moment of diagnosis, el $36 \%$ of the cases were local, $34 \%$ were regional and $23 \%$ disseminated. Crude, standardized and truncated (35-64 years) incidence rates, during 1980 to 1989 were de 8.2, 6.2 and 13.7 cases $\times 105$ women respectively. According to the cumulative rate, 1 in 150 women will have this cancer by age 74 . No increase of the trend has been found, however, a significative subincidence has been observed in rural areas. 5-years survival rates is $48.5 \%$ and was significantly associated with stage at diagnosis, but age was not.

Conclusion: From an international viewpoint, Gerona is a low incident area for this tumour and no rising trend has been found. The subincidence in rural areas may suggest a different exposition to risk factors, although certain aspects such as accessibility to health care services, the defining of rural and urban areas and the existence of migrations should be considered. Finally, the lower survival rate with regard to other countries could be attributed to the presence of more advanced stages, which emphasizes the importance that the early diagnosis of this tumour has.

KEY WORDS: Invasive Cervical Cancer, Population Based Cancer Records. Incidence. Survival. 


\section{INTRODUCCIÓN}

El cáncer de cérvix es el segundo tumor más frecuente entre las mujeres a nivel mundial, aunque su incidencia presenta importantes variaciones internacionales. Así, en la mayor parte de países no industrializados es el tumor femenino más frecuente, mientras que en los países occidentales desarrollados ocupa el décimo lugar ${ }^{1.2}$. En España, según los datos de diferentes registros poblacionales de cáncer, este tumor presenta comparativamente una baja incidencia ${ }^{3}$.

Estas diferencias en los patrones de afectación constituyeron el punto de partida de estudios analíticos que pusieron en evidencia una relación causal con el Virus del Papiloma Humano (VPH), quedando factores de riesgo clásicos (comportamiento sexual de la mujer y su pareja o estatus socioeconómico) como indicadores de exposición a dicho virus ${ }^{4-6}$. Otros factores de riesgo considerados han sido el tabaquismo, el consumo de anticonceptivos orales y ciertas deficiencias nutricionales, aunque todavía no está claro si estos actúan independientemente del HPV ?

La importancia epidemiológica del cáncer de cérvix se centra en su tipo histológico más frecuente, el carcinoma escamoso, que tiene su origen en el epitelio de transición del cuello uterino. Su larga evolución (displasia-carcinoma in situ-carcinoma invasor), su accesibilidad anatómica y la disponibilidad de técnicas de citología exfoliativa permiten su detección precoz con facilidad, habiéndose demostrado la efectividad del cribado a partir de estudios de incidencia y mortalidad en países nórdicos ${ }^{8}$.

En la mayoría de países industrializados este tumor ha presentado una tendencia descendente en las últimas décadas ${ }^{9-11}$, aunque a partir de los años 70 , se ha observado en alguno de ellos un incremento de su incidencia y mortalidad entre mujeres jóvenes, existiendo cierta controversia sobre este fenómeno ${ }^{10,12-14}$.

Los registros de cáncer poblacionales son un instrumento fundamental que nos permite conocer su impacto en la población y los posibles factores asociados a su aparición, así como realizar una evaluación de la efectividad de las medidas aplicadas en la lucha contra csta cnfermedad. El objetivo de este trabajo es realizar un estudio descriptivo del impacto del cáncer invasor de cérvix en el Area Sanitaria de Gerona, así como identificar las posibles diferencias exis tentes en su distribución demográfica utilizando como fuente de información los datos recogidos por el Registro Poblacional de Cáncer de Gerona (RCG).

\section{MATERIAL Y MÉTODOS}

El Registro Poblacional de Cáncer de Gerona recoge, desde 1980, todos los casos incidentes de tumores ginecológicos y de mama aparecidos entre las mujeres residentes en el Area Sanitaria constituida por seis comarcas (Alt Empordà, Baix Empordà, Garrotxa, Gironés, Pla de l'Estany y La Selva) ${ }^{15}$. Según el censo de 1986, la población cubierta por el Registro era de 227.349 mujeres. De estas, el $24 \%$ nacieron fuera de Cataluña.

La información recogida comprende variables sociodemográficas, variables relativas al tumor y variables de seguimiento del paciente. Los tumores se codifican según la Clasificación Internacional de Enfermedades para Oncología (CIEO) ${ }^{16}$. Para la clasificación de la extensión tumoral en el momento del diagnóstico se utiliza una versión simplificada de diferentes clasificaciones (FIGO 1985, TNM-UICC 1982). Se considera «local» al tumor que solo afecta un órgano, «regional» cuando aparece invasión ganglionar o de órganos vecinos y «diseminado» si se observan metástasis. Los criterios de definición de segunda neoplasia son los recomendados por la International Agency for Research in Cancer (IARC): localización del tumor en órganos diferentes (excluyéndose las metástasis); y localización de más de un tumor con distinto tipo histológico en un mismo órgano ${ }^{17}$.

El porcentaje de verificación histológica para el cáncer invasor de cérvix es del $98,4 \%$, no existiendo ningún caso obtenido exclusivamente a través del Boletín Estadístico de Defunción (porcentaje de DCO). El porcentaje de DCO se refiere exclusivamente al período $1985-89$, ya que es a partir de 1985 cuando se dispone de información de los Boletines de Defunción.

Fl período de análisis abarca 10 años (19801989). La población correspondiente a cada año del estudio y su distribución por edades se ha estimado por interpolación del incremento medio anual ponderado entre el censo de 1981 y el padrón de 1986. Las variables estudiadas son: edad 
media de presentación, tipo histológico, aparición de segundas neoplasias, distribución de casos según lugar de origen y extensión del tumor en el momento del diagnostico.

A partir de los datos de incidencia se han calculado Tasas Brutas, Ajustadas, Truncadas (entre 35 y 64 años), expresadas todas ellas como número de casos por $10^{5}$ mujeres y Tasa Acumuladas (entre 0 y 74 años), expresadas como porcentajes. El ajuste de tasas se ha realizado por el método directo, tomando como referencia la población estándar mundial ${ }^{18}$. Estos cálculos se han realizado para los períodos 80-89, 80-84 y 85-89. Así mismo, se ha llevado a cabo un análisis de tendencia, utilizando un modelo de regresión que asume una distribución de Poisson ${ }^{19.20}$, estimándose un porcentaje de cambio anual a partir del coeficiente de la variable «año» en el modelo ajustado, que incluía la variable «grupo de edad». Dicho análisis comprende solo el período 1985-89.

El estudio de la distribución demográfica se ha realizado mediante el cálculo de la Razón de Incidencia Estandarizada (RIE) y de su intervalo de confianza al $95 \%$ (IC95\%) ${ }^{21}$, utilizando como referencia las tasas de incidencia por grupos de edad del Area Sanitaria. Se han considerado como «urbanas» las poblaciones mayores de 10.000 habitantes y como «rurales» las menores de $10.000^{22}$.

La supervivencia observada así como la relativa se han calculado de forma global y en función de las variables edad y extensión en el momento del diagnóstico. El método utilizado para el cálculo de las curvas de supervivencia ha sido el de Kaplan-Meier. La comparación de curvas según diferentes variables se ha realizado mediante el log-rank test y así mismo se ha utilizado el método de los riesgos proporcionales de Cox para estudiar el efecto simultáneo de diferentes variables sobre la supervivencia ${ }^{23}$.

\section{RESULTADOS}

A lo largo del período estudiado (1980-89) se han registrado 185 casos incidentes de cáncer invasor de cérvix, con una edad media de presentación de $57 \pm 14$ años. El tipo histológico más frecuente corresponde al carcinoma escamoso, con 156 casos ( $84 \%$ del total), seguido del adenocarcinoma con 11 casos (6\%). El tipo carcinoma, sin otra especificación, comprende el $6 \%$. Un cuarto grupo reúne una serie de tumores de rara presentación (carcinoma adenoescamoso, endometrioide y carcinosarcoma) con frecuencias relativas muy bajas (2\%). En un $2 \%$ de los casos se desconocía la histología. En 5 casos (2.7\%) aparece una segunda neoplasia localizada en ovario, piel, cerebro, mama y endometrio respectivamente. En relación a la distribución de casos según la región de origen, aproximadamente el $51 \%$ corresponden a las mujeres nacidas en Cataluña, y el $32 \%$ a las mujeres procedentes del resto de España. Un 10\% de casos corresponde a mujeres extranjeras y en un $7 \%$ no constaba el lugar de origen (Tabla 1).

En el momento del diagnóstico, el 36\% de los casos eran locales, el $34 \%$ regionales y el $23 \%$ eran diseminados. En el $7 \%$ de los casos no constaba la extensión tumoral.

Las diferentes tasas de incidencia por este tumor obtenidas en el período estudiado en el Area Sanitaria quedan recogidas en la tabla 2. En la figura 1 se presenta una comparación internacional de las Tasas Ajustadas de incidencia. En la tabla 3 se presentan, en forma de porcentaje de incremento medio anual, los resultados del aná-

Tabla 1

Incidencia del cáncer de cérvix. Área sanitaria. Distribución según lugar de nacimiento

\begin{tabular}{|lrrrrrr|}
\hline & \multicolumn{2}{c}{$1980-84$} & \multicolumn{2}{c|}{$1985-89$} & \multicolumn{2}{c|}{$1980-89$} \\
\hline Cataluña & $\mathbf{N}$ & $\%$ & $\mathbf{N}$ & $\%$ & $\mathbf{N}$ & $\%$ \\
Resto de España & 43 & 51 & 51 & 50 & 94 & 51 \\
Extranjeros & 23 & 27 & 36 & 36 & 59 & 32 \\
Desconocido & 9 & 11 & 10 & 10 & 19 & 10 \\
Total & 9 & 11 & 4 & 4 & 13 & 7 \\
\hline
\end{tabular}

Fuente: Registro Poblacional de Cáncer de Girona. 
Tabla 2

Tasas de incidencia del cáncer de cérvix. Área sanitaria

\begin{tabular}{|cccccccccccccccccccc|}
\hline & & & & & & & & & & & & TB & T.A & T.TR & T.AC \\
Período & Casos & $20-$ & $25-$ & $30-$ & $35-$ & $40-$ & $45-$ & $50-$ & $55-$ & $60-$ & $65-$ & $70-$ & $75-$ & $80-$ & $85+$ & & Mund $35-64$ & $0-74$ \\
\hline $1980-84$ & 84 & 0,0 & 0,0 & 8,3 & 6,4 & 13,6 & 9,0 & 13,3 & 23,3 & 17,7 & 17,9 & 15,3 & 14,4 & 14,5 & 21,1 & 7,8 & 5,8 & 13,0 & 0,62 \\
$1985-89$ & 101 & 1,2 & 2,3 & 7,7 & 6,9 & 16,2 & 20,0 & 16,3 & 11,6 & 20,4 & 28,3 & 15,0 & 23,3 & 8,5 & 9,3 & 8,9 & 6,9 & 15,1 & 0,73 \\
$1980-89$ & 185 & 0,6 & 1,2 & 7,7 & 6,2 & 13,8 & 15,4 & 14,8 & 16,6 & 18,1 & 22,6 & 15,0 & 18,2 & 10,6 & 13,9 & 8,2 & 6,2 & 13,7 & 0,66 \\
\hline
\end{tabular}

TB: T. Bruta / T.A: T. Ajustada Mundial / T.TR: T. Truncada (35-64 años) / TAC: T. Acumulada (0-74 años)

Fuente: Registro Poblacional de Cáncer de Girona.

Figura 1

Comparación Internacional de la incidencia por cáncer de cérvix. Tasas ajustadas 1983-1987

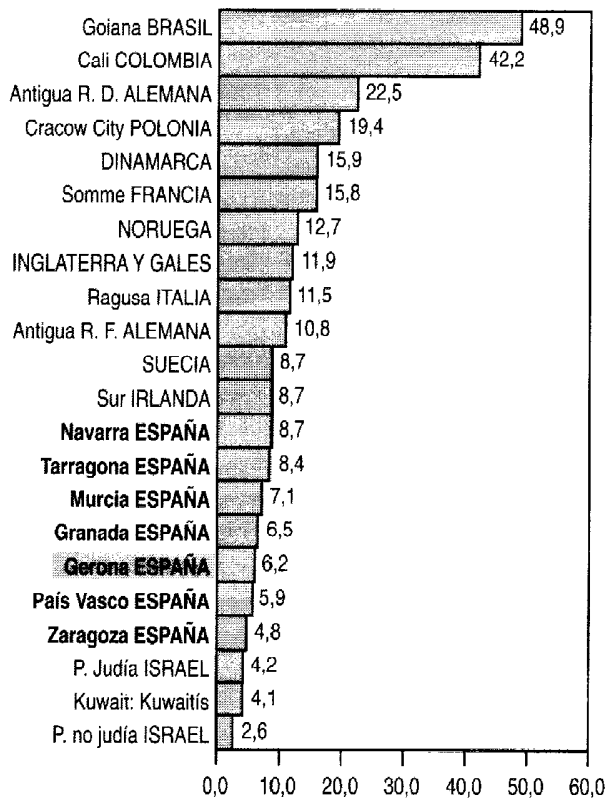

Tabla 3

Tendencia de la incidencia del cáncer de céryix. Área sanitaria 1985-1989

\begin{tabular}{|cccc|}
\hline & Casos & Cambio anual (\%) & IC 95\% \\
\hline Global & 101 & 2.1 & $-11: 17.2$ \\
$35-64$ años & 58 & 4.2 & $-13: 25.1$ \\
$>64$ años & 34 & -3.9 & $-24: 21.7$ \\
\hline
\end{tabular}

Fuente: Registro Poblacional de Cáncer de Girona.

lisis de tendencia de la incidencia de este tumor, entre 1985 y 1989 . Se ha analizado la incidencia total, y separada por grandes grupos de edad (3564 años $y \geq 65$ años). Aunque se observan incrementos en el total y en el grupo de 35-64 años, estos no son significativos. En relación a la distribución demográfica, no se aprecian diferencias significativas en las zonas urbanas, con una RIE de 117.0 (90.7:140.7); por el contrario se observa una subincidencia significativa en zonas rurales con una RIE de 77.8 (61.3:98.7).

La tabla 4 presenta la probabilidad de supervivencia observada y relativa en diferentes momentos del seguimiento, y la mediana de super-

Tabla 4

Supervivencia del cáncer de cérvix. Área sanitaria 1980-1989

\begin{tabular}{|c|c|c|c|c|c|c|c|c|c|c|c|}
\hline & \multicolumn{3}{|c|}{ Observada (\%) } & \multirow[b]{2}{*}{$5 \mathrm{a}}$. & \multirow[b]{2}{*}{$10 \mathrm{a}}$. & \multirow[b]{2}{*}{ mediana* } & \multicolumn{5}{|c|}{ Relativa (\%) } \\
\hline & Casos & 1 a. & $3 \mathrm{a}$. & & & & $1 \mathrm{a}$. & $3 \mathrm{a}$. & $5 \mathrm{a}$. & $10 \mathrm{a}$. & mediana* \\
\hline TOTAL & 185 & 80 & 57 & 46 & 36 & 48 & 81 & 59 & 49 & 40 & 54 \\
\hline EDAD & & & & & & & & & & & \\
\hline 35-64 años & 125 & 83 & 62 & 50 & 38 & 57 & 83 & 62 & 50 & 40 & 60 \\
\hline$>64$ años & 59 & 76 & 47 & 39 & - & 27 & 78 & 51 & 44 & - & 36 \\
\hline \multicolumn{12}{|c|}{ EXTENSION } \\
\hline Local & 66 & 94 & 84 & 70 & 64 & $>130$ & 94 & 82 & 71 & 67 & $>102$ \\
\hline Regional & 62 & 80 & 52 & 42 & - & 42 & 81 & 54 & 44 & - & 90 \\
\hline Diseminado & 43 & 63 & 26 & 21 & - & 15 & 64 & 26 & 22 & - & 12 \\
\hline
\end{tabular}

(* meses)

Fuente: Registro Poblacional de Cáncer de Gerona. 
vivencia (expresada en meses). Estos cálculos se han realizado para la totalidad de los casos, así como para subgrupos definidos en función de la edad y la extensión en el momento del diagnostico. La figura 2 presenta la curva de supervivencia para el total de los casos. Si comparamos las curvas de supervivencia en función de la edad (figura 3), las pacientes diagnosticadas después de los 65 años presentan peor supervivencia que las más jóvenes, aunque esta diferencia no alcanza significación estadística $(p=0.09)$. La figura 4 presenta la probabilidad de supervivencia en función del estadio en el momento del diagnostico. Las diferencias entre las curvas son marcadas y estadísticamente significativas $(\mathrm{p}<0.001)$. En relación a los resultados del estudio de factores pronósticos, hay que señalar que solo la extensión en el momento del diagnos-

Figura 2

Supervivencia del cáncer de cérvix. Área Sanitaria de Gerona

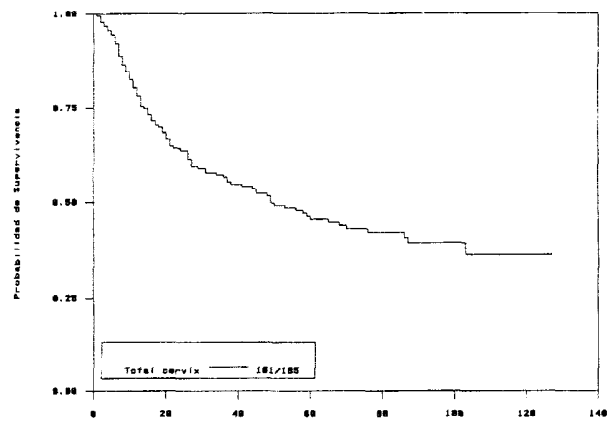

Figura 3

Supervivencia del cáncer de cérvix por grupos de edad, Área sanitaria de Gerona.

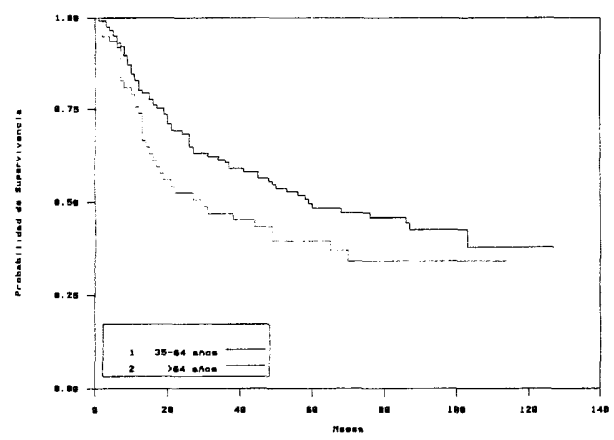

tico influye en la supervivencia. En la tabla 5 se detalla únicamente esta variable, ya que la edad carecía de significación estadística.

\section{DISCUSIÓN}

$\mathrm{Al}$ analizar los resultados obtenidos en este estudio observamos que, tanto la edad media de presentación de este tumor como la distribución de tipos histológicos, son similares en Gerona a las descritas en la literatura internacional ${ }^{24,25}$. Por el contrario, llaman la atención las diferencias en la distribución de estadíos frente a los datos aportados por otros países ${ }^{26,27}$, observándose una menor proporción de estadíos locales y una mayor de los estadios diseminados. Esto sugiere la existencia de un retraso en el diagnóstico en Gerona respecto a otros países industrializados.

Otro aspecto a destacar es el elevado porcentaje de mujeres inmigrantes entre los casos de cérvix recogidos en Gerona. En este sentido, un estudio llevado a cabo recientemente descubrió

Tabla 5

Supervivencia del cáncer de cérvix. Área sanitaria 1980-1989. Factores pronósticos

\begin{tabular}{|cccc|}
\hline & Riesgo & IC 95\% & $P$ \\
\hline Local & 1 & & \\
Regional & 2.13 & $1.2-3.75$ & 0.009 \\
Diseminado & 5.06 & $2.90-8.80$ & $<0.001$ \\
\hline
\end{tabular}

Fuente: Registro Poblacional de Cáncer de Girona.

Figura 4

Supervivencia del cáncer de cérvix según estadio. Área sanitaria de Gerona.

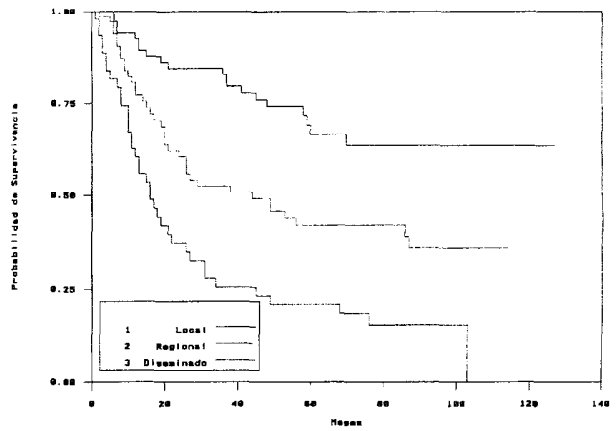


una sobreincidencia significativa de cáncer de cérvix en las mujeres procedentes de regiones del sur de España en relación a las nacidas en la provincia de Gerona. Dichas mujeres presentaban estadíos más avanzados en el momento del diagnóstico. Al mismo tiempo, se observó una menor proporción de mujeres inmigrantes entre las que presentaban un diagnóstico de carcinoma in situ; por lo que esta sobreincidencia se ha atribuido fundamentalmente a una menor participación en el cribado de cáncer de cérvix ${ }^{28}$.

La distribución internacional de la incidencia del cáncer de cérvix presenta importantes variaciones, observándose las tasas más elevadas en Latinoamérica y Sureste Asiático, mientras que las tasas más bajas aparecen en Oriente Medio, Finlandia y España ${ }^{29}$. Por otra parte, a pesar de la tendencia descendente tanto de la incidencia como de la mortalidad por este tumor en la mayoría de países industrializados ${ }^{30-32}$, en las últimas décadas se han descrito aumentos de las mismas en mujeres jóvenes ${ }^{31} \mathrm{de}$ cstos países ${ }^{31-35}$. Aunque existe cierta evidencia de que la mortalidad por cáncer de cérvix ha aumentado en España ${ }^{30.33}$, no se ha observado el incremento entre las mujeres jóvenes señalado anteriormente ${ }^{36}$.

Las tasas de incidencia observadas en Gerona la sitúan en los últimos lugares de la clasificación internacional. En relación a España ocupa un lugar medio entre las comunidades con registros poblacionales de cáncer ${ }^{3,37}$. Si se comparan los dos subperíodos estudiados (80-84 y 85-89) se puede observar un ligero aumento de las tasas en el segundo período respecto al primero, así como un afectación de grupos de edad más jóvenes. No obstante, el estudio de la tendencia no muestra un incremento, ya que las variaciones advertidas tanto para el total de casos, como para los grupos de edad no son significativas. El descenso de la incidencia entre las mujeres de edades avanzadas, aunque no es significativo, podría deberse en parte a un diagnóstico menos exhaustivo del tumor. Las tasas truncadas (35-64 años) son claramente superiores a las ajustadas, lo que nos indicaría un predominio en las edades medias. Las tasas acumuladas (0-74 años) indican una probabilidad de padecer este tumor del $0,66 \%$, lo que supone que una de cada 150 mujeres lo padecerán antes de los 75 años.

El estudio demográfico muestra una subincidencia significativa en zonas rurales respecto al Area Sanitaria, hecho que podría estar relacio- nado con una diferente exposición a los factores de riesgo. No obstante, hay que interpretar estos datos con precaución ya que, a pesar de que pueden reflejar diferencias reales, hay que tener en cuenta aspectos como la accesibilidad a los servicios sanitarios y la calidad de los mismos en las diferentes zonas, así como el criterio elegido para la definición de medio urbano y rural. Por otra parte, la presencia de movimientos poblacionales migratorios puede provocar una afluencia de poblaciones con diferente grado de incidencia. En el caso de Gerona, las zonas urbanas tienen una mayor presencia de población inmigrante entre la que se ha observado una incidencia mayor de este tumor.

La tasa observada de supervivencia de los casos de cáncer de cérvix registrados en el Area Sanitaria de Gerona fue del $80 \%$ al año, y del $46 \%$ a $\operatorname{los} 5$ años. La supervivencia relativa presenta tasas ligeramente superiores, con una mediana de 54 meses. En relación a la edad, las diferencias observadas no son significativas. La extensión del tumor en el momento del diagnóstico aparece inversamente relacionada con la probabilidad de supervivencia, siendo dicha probabilidad a los 5 años del $22 \%$ en el caso de tumores diseminados (con solo 12 meses de mediana de supervivencia). De hecho, esta es la única variable que presenta significación estadística en el análisis de factores pronósticos. Así, las pacientes con un tumor regional tienen un riesgo de muerte 2 veces superior a las que presentan un tumor local, mientras que si el tumor es diseminado el riesgo de muerte es 5 veces superior.

Si comparamos las tasas de Gerona con las presentadas en otros estudios americanos y europeos $^{27,28}$, observamos que las probabilidades de supervivencia de Gerona son más bajas. Así, la probabilidad de supervivencia relativa a los 5 años en la población blanca de Estados Unidos fue del $67 \%$ y la media europea occidental del $54 \%$. En este sentido, hay que tener en cuenta que la serie americana presenta una mayor proporción de estadíos locales que la de Gerona, y que incluye una combinación de datos poblacionales y hospitalarios, hechos que podrían explicar la mejor supervivencia observada. En relación a la media europea hay que señalar que esta incluye países donde desde hace tiempo se han llevado a cabo programas de cribado, lo que favorece la detección del tumor en estadíos precoces y, por tanto, con mayor probabilidad de supervivencia. 
En términos generales se puede concluir que en relación con la situación internacional, Gerona es un área de baja incidencia de cáncer de cérvix, no observándose una tendencia ascendente de la misma aunque se aprecia una subincidencia observada en zonas rurales. La menor supervivencia observada en comparación con otros países industrializados podría atribuirse a la presencia de estadíos más desfavorables, lo que pone de relieve la importancia de la detección precoz de este tumor, orientada fundamentalmente a los grupos de alto riesgo, que son probablemente los que presentan una menor participación en esta prueba. Por otra parte, las posibilidades de prevención secundaria podrían ampliarse, en el futuro, complementando la citología con algún test virológico, dada la reciente confirmación de que la presencia de VPH en material exfoliado es un buen predictor del riesgo de progresión de lesiones preneoplásicas ${ }^{39,40}$. En términos de prevención primaria, actualmente está en fase de estudio el desarrollo de vacunas frente al HPV ${ }^{41}$.

\section{AGRADECIMIENTOS}

Al Doctor Victor Moreno por su colaboración en el análisis estadístico de los datos y por los comentarios a una versión previa de este artículo.

\section{BIBLIOGRAFÍA}

1. Parkin DM, Laara E, Muir CS. Estimates of the worldwide frequency of sixteen major cancers in 1980. Int J Cancer 1988; 41: 184-189.

2. Parkin DM, Pisani P, Ferlay J. Estimates of the worldwide incidence of eighteen majors cancers in 1985. Int J Cancer 1993: 54: 594-606.

3. Ministerio de Sanidad y Consumo. Cáncer en España. Madrid: Ministerio de Sanidad y Consumo, 1993.

4. Bosch FX, Muñoz N, De Sanjosé S, Izarzugaza I, Gili M, Viladiu P et al. Risk Factors for Cervical cancer in Colombia and in Spain. Int $\mathbf{J}$ Cancer 1992; 52: 750-758.

5. Bosch FX, Muñoz N, Shah KV, Meheus A. Second International Workshop on the Epidemiology of Cervical cancer and Human Papillomavirus. Int J Cancer 1992; 52: 171-173.

6. Bosch FX, Muñoz N, De Sanjosé S, Eleuf Neto J, Orfila J, Walboomers $\mathbf{J}$ et al. What is relevant in Cervical Carcinogenesis other than HPV? En:
Monsonego, editor. Screening of cervical cancer for whom, why and how? Proceeding as of the 2nd International Congress of Papillomavirus in Human Pathology; 1994 Abril 7; Paris: Eurogin Scientific Publications, 1995.

7. Muñoz N, Bosch FX, Shah KV, Meheus A, editores. The Epidemiology of Human Papillomavirus and Cervical Cancer. Lyon: IARC Scientific Publications n. ${ }^{\circ} 119,1992$.

8. Hakama M, Magnus K, Petterson F, Storm H, Tulinius $H$. Efect of organized screening on the risk of cervical cancer in the nordic countries. En: Millet AB, Hakama M. Day NE, Chamberlain J, Prorock PC, editores. Cancer screening. Cambridge: Cambridge University Press, 1991: 153-162.

9. Jensen O, Esteve J, Moller H, Renald H. Cancer in the European Community and its Member States. Eur J Cancer 1990; 26: 1167-1256.

10. Devesa S, Silverman D, Young JL, Pollack ES, Brown CC, Horm JW et al. Cancer Incidence and Mortality Trends Among Whites in the United States, 1947-84. J. Natl Cancer I 1987; 79(4): 701-745.

11. Franceschi S, Levi F, La Vecchia C, Lucchini F, Negri E. Comparison of Cancer Mortality in Major European Areas 1960-89. Eur J Cancer Prev 1994; 3: 145-206.

12. Cuzick J, Boyle P. Trends in cervix cancer mortality. Cancer Sur 1988; 7(3): 417-438.

13. La Vecchia C, Luchini F, Negri E Boyle P, Maisoneuve $P$, Levi P. Trends of cancer mortality in Europe, 1955-1989: III, Breast and Genital Sites. Eur J Cancer 1992; 28: 927-988.

14. Macgergor E, Campbell M, Mann E, Swanson K. Screening for cervical intraepitelial neoplasia in north east Scotland shows fall in incidence and mortality from invasive cancer with concomitant rise in preinvasive disease. Brit Med J 1994; 308: 1407-1411.

15. Viladiu $\mathrm{P}$, Izquierdo A, Beltrán $\mathrm{M}$, Bosch $\mathrm{FX}$, Moreno V. Epidemiología del cáncer ginecológico y de mama en el Área Sanitaria de Gerona. Registre del Cáncer de Gerona. Gerona: Asociación Española contra el Cáncer, 1995.

16. CIE-O. Clasificación Intnernacional de Enfermedades para Oncología. Washington: Organización Panamericana de la Salud. Publicación Científica n. ${ }^{\circ} 345,1977$.

17. Jensen OM, Parkin DM, Maclennan, R, Muir CS, Skeet RG. Cancer Registration: Principles and Methods. Lyon: IARC Scientific Publications n. ${ }^{\circ}$ 95, 1991.

18. Rates and Rate Standardization. En: Breslow N and Day NE. Statistical methods in cancer rese- 
arch. Vol. II. Lyon: IARC Scientific Publications n. ${ }^{\circ} 82,1993$.

19. Fitting models to grouped data. En: Breslow NE and Day NE. Statistical methods in cancer research. Vol II. Lyon: IARC Scientific Publications n. ${ }^{\circ} 82,1987: 120-176$.

20. Atkin M, Anderson C, Francis B, Hinde J. Statistical modelling with GLIM. Oxford: Oxford University Press, 1989: 217-255.

21. Jensen OM, Parkin DM, Maclennan R, Muir CS, Skeet RG, editores. Cancer Registration: Principles and Methods. Lyon: IARC Scientific Publications n. ${ }^{\circ}$ 95, 1991.

22. Roginski C. Comparison of urban and rural incidence data. En: Muir C, Waterhouse J, Mack T, Powell J, Whelan S, editores. Cancer Incidence in Five Continents. Vol V. Lyon: IARC Scientific Publications n. ${ }^{\circ} 88,1987$.

23. Techniques d'analyse de la survie. En: Estève L, Benhamou E, Raymond L. Méthodes statistiques en épidémiologie descriptive. Paris: INSERM, 1993.

24. Ulmer HU, Hossfeld DK, Love RR. Cancers of the Uterine Cervix and Endometrium and Gestational Trophoblastic Disease. En: Love RR, editor. Manual of Clinical Oncology. Ginebra: UICC, 1994.

25. Williams C. Ovarian and cervical cancer. En: Mead GM, editor. Current issues in cancer. Londres: BMJ Publishing Group, 1992.

26. Ries LAG, Miller BA, Hankey BF, Kosary CL, Harras A, Edwards BK, editores. SEER Cancer Statistics Review 1973-1991: Tables and Graphs, National Cancer Institute. Bethesda (MD): NIH Publication n. ${ }^{\circ}$ 94-2789, 1994.

27. Population Screening for Cervical Cancer in the Netherlands. A Report by the Evaluation Committee. Int J Epidemiol 1989; 18(4): 775-781.

28. Borràs JM, Sánchez V, Izquierdo A, Moreno V, Viladiu P. Cervical cancer: incidence and survival in migrants within Spain. J Epidemiol Commun Health 1995; 49: 153-157.

29. Parkin DM, Muir CS, Whelan SL, Gao Y-T, Ferlan J, Powel J, editores. Cancer Incidence in Five Continents. Vol VI. Lyon: AIRC Scientific Publications n. ${ }^{\circ}$ 120, 1992.

30. Jensen O, Esteve J, Moller H, Renald H. Cancer in the European Community and its Member States. Eur J Cancer 1990; 26: 1167-1256.
31. Devesa S, Silverman D, Young Л, Pollack ES, Brown CC, Horm JW et al. Cancer Incidence and Mortality Trends Among Whites in the United States, 1947-84. J Nat Cancer I 1987; 79(4): 701-745.

32. Franceschi S, Levi F, La Vecchia C, Lucchini F, Negri E. Comparison of Cancer Mortality in Major European Areas 1960-89. Eur J Cancer Prev 1994; 3: 145-206.

33. Cuzick J, Boyle P. Trends in cervix cancer mortality. Cancer Surv 1988; 7(3); 417-438.

34. La Vecchia C, Luchini F, Negri E Boyle P, Maisoneuve P, Levi P. Trends of cancer mortality in Europe, 1955-1989: III, Breast and Genital Sites. Eur J Cancer 1992; 28: 927-988.

35. Macgergor E, Campbell M, Mann E, Swanson K. Screening for cervical intraepitelial neoplasia in north east Scotland shows fall in incidence and mortality from invasive cancer with concomitant rise in preinvasive disease. Brit Med J 1994; 308: 1407-1411.

36. Sánchez MV, Izquierdo A, Beltrán M, Bosch José FX, Viladiu Quemada P. Tendencias temporales de la mortalidad por cáncer de cérvix en Cataluña 1975-1992: Análisis del Boletín Estadístico de Defunción y del Kegistro de Cáncer de Gerona. Gac Sanit 1996; 10: 67-72.

37. Martos MC, Tomás C, Gómez L. Incidencia del cáncer genital femenino en la provincia de Zaragoza 1975-1984. Med Clin (Barc) 1992; 99 : 410-413.

38. Berrino F, Sant M, Verdecchia A, Capocaccia R, Hakulinen T, Estève J, editores. Survival of Cancer Patients in Europe. The EUROCARE Study. Lyon: IARC Scientific Publications n. ${ }^{\circ}$ 132, 1995.

39. Muñoz N, Bosch FX. HPV and cervical neoplasia. Review of case-control studies. En: Muñoz N, Bosch FX, Shah KV, Meheus A, editores. The epidemiology of cervical cancer and human papillomavirus. Lyon: IARC Scientific Publications n. ${ }^{\circ} 119,1992$.

40. Day Ne, Veral V. Screening for cervical cancer: Is there a place for incorporating test for the human papillomavirus? En: Muñoz N, Bosch FX, Shah $\mathrm{KV}$, Meheus A, editores. The epidemiology of cervical cancer and human papillomavirus. Lyon: IARC Scientific Publications n. . 119, 1992.

41. IARC and Fondation Marcel Merieux. HPV vaccines and their potential use in the prevention and treatment of cervical neoplasia. Lyon: Foundation Marcel Merieux, 1994. 